\title{
Time-resolved reflectivity and Raman studies of the interplay of enigmatic orders in $\mathrm{Mo}_{8} \mathrm{O}_{23}$
}

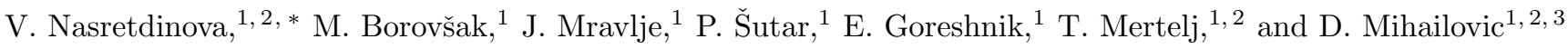 \\ ${ }^{1}$ Jozef Stefan Institute, Jamova 39, 1000 Ljubljana, Slovenia \\ ${ }^{2}$ Center of Excellence on Nanoscience and Nanotechnology Nanocenter \\ (CENN Nanocenter), Jamova 39, 1000 Ljubljana, Slovenia \\ ${ }^{3}$ Faculty of Mathematics and Physics, University of Ljubljana, Jadranska 19, 1000 Ljubljana, Slovenia
}

(Dated: November 11, 2019)

\begin{abstract}
Monoclinic semi-metallic $\mathrm{Mo}_{8} \mathrm{O}_{23}$ belongs to a multifunctional series of compounds showing multiple ordering phenomena that have not achieved much attention till now. Previous X-rays studies of this compound have revealed an incommensurate ordering transition at $T_{\mathrm{IC}} \sim 350 \mathrm{~K}$, followed by a structural transition to commensurate order at $T_{\mathrm{IC}-\mathrm{C}}=285 \mathrm{~K}$. In addition, an enigmatic resistance maximum is observed at $T_{\mathrm{el}} \sim 150 \mathrm{~K}$, whose origin has so far proved elusive. Aiming to disentangle these multiple orders we use the polarized transient optical spectroscopy supplemented by Raman spectroscopy to study the electronic relaxation dynamics and lattice vibrational modes in $\mathrm{Mo}_{8} \mathrm{O}_{23}$ single crystals. Remarkably, both the coherent vibrational mode response and single particle response display extrema of damping/relaxation times close to $T_{\mathrm{el}}$ with the concurrent appearance of new coherent vibrational modes and a characteristic polarization asymmetry which saturates below $T_{\text {el }}$. The single-particle relaxation data analysis shows the appearance of a temperature-independent gap in the electronic excitation spectrum below $T_{\mathrm{IC}}$ and additional temperature-dependent gap opening near $T_{\mathrm{el}}$. Concurrently, a low frequency vibrational mode shows anomalous softening around $T_{\mathrm{m}} \sim 200 \mathrm{~K}$, far below $T_{\mathrm{IC}-\mathrm{C}}$ and $T_{\mathrm{IC}}$. The observations are interpreted in terms of the appearance of a hidden gapped state below $T_{\mathrm{el}}$ that has so far eluded detection by structural analyses.
\end{abstract}

\section{INTRODUCTION}

Low-dimensional molybdenum oxide $\mathrm{Mo}_{8} \mathrm{O}_{23}$ belongs to the family of $\mathrm{MoO}_{3-x}$ suboxides (Magneli phases), which have been studied as perspective charge storage or battery materials ${ }^{113}$. More recently, the ideas of memory devices based on electrical switching/topotactic transitions in molybdenum suboxides have been discussed $\frac{415}{}$. In spite of increased recent interest in their functional properties, the phase diagrams, transient and even equilibrium optical properties have not been systematically studied for many molybdenum suboxides.

In the case of $\mathrm{Mo}_{8} \mathrm{O}_{23}$, structura $[\sqrt[6]{9}$ and transport studies $\sqrt{6}$ suggest that following the emergence of a hightemperature incommensurate order at $T_{\mathrm{IC}} \sim 350 \mathrm{~K}$, an intriguing and unusual $\mathrm{C}_{2}$-and inversion symmetry breaking commensurate charge density wave (CDW) state (see Fig. 1) occurs below $T_{\mathrm{IC}-\mathrm{C}}=285 \mathrm{~K}$. Curiously, the resistivity shows an anomalous peak around $T_{\mathrm{el}} \sim 150 \mathrm{~K}$ that cannot be understood in terms of the high-temperature CDW ordering. Thus the nature of the low-temperature $\left(T<T_{\mathrm{IC}-\mathrm{C}}\right)$ phase seems to be more complicated than anticipated, possibly with multiple orders present.

CDW's that break not only translational but also rotational in-plane symmetries of the underlying lattice are $\operatorname{rar} \mathrm{e}^{10}$. On the other hand, the antiferrodistortive structural transitions driven by soft zone boundary phonon with displacements described by octahedral rotation are common phenomena in the insulators and could produce incommensurate phase ${ }^{111}$ due to the competition of short-range forces 12 . Such a scenario has been proposed

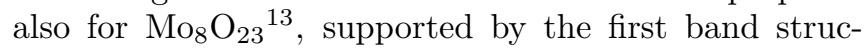

ture consideration ${ }^{14}$ that found only a weak band dispersion along the commensurate wave vector $q_{\mathrm{C}}=(0,0.5,0)$. The increase of $T_{\mathrm{IC}-\mathrm{C}}$ under pressure ${ }^{15}$ is also common to the antiferrodistortive transitions $\frac{16 \mid 17}{10}$ and not expected for a CDW-type nesting instability $\underline{18}$, although some exceptions were reported 19 .

Yet room-temperature STM atomic-resolution images of $\mathrm{Mo}_{8} \mathrm{O}_{23}$ [cleaved (010) surface] demonstrate charge modulation ${ }^{20}$, characteristic for CDW's 18 . Moreover, the density-functional-theory (DFT) ab-initio calculations (to be reported elsewhere ${ }^{21}$ ) show enhancement of the Lindhard susceptibility in a broad range of wave vectors, much like in $\mathrm{TiSe}_{2}{ }^{22}$, and give some support for the nesting-driven CDW scenario for both, the commensurate and incommensurate phases.

In the present case the equilibrium-state experimental techniques may be insufficient to completely disentangle structural and electronic orders and the origin of the underlying interactions and need to be complemented by non-equilibrium techniques. Among the latter the ultrafast time-resolved optical spectroscopy is useful in elucidating the single particle and collective excitations in relation to the various order parameters by separating their timescales and may even help to detect electronic gaps $\$ 23$. Moreover, the probe-polarization resolved pump-probe studies can help to identify different components of the transient response in noncentrosymmetric materials ${ }^{30}$ or different orders in correlated systems such as pseudogaps and superconducting gaps in the cuprates 31 and organic superconductors 32 34

Here we present a probe-polarization-resolved transient reflectivity study of the phase diagram and particularly the low-temperature anomalous CDW state in 


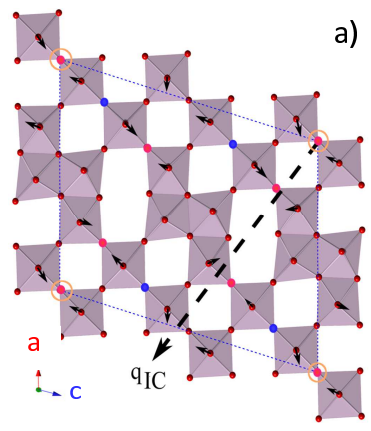

c)

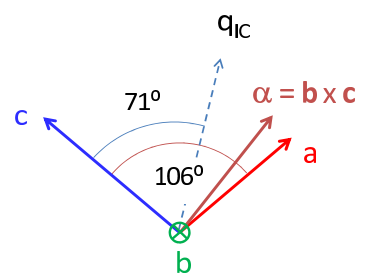

b)

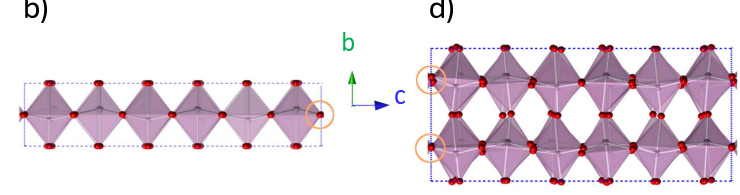

Figure 1. The crystal structure of $\mathrm{Mo}_{8} \mathrm{O}_{23}$ after ref.617. The fine-dashed lines denote the unit cells and the encircled atoms the inversion centers of the high- $T$ structure. a) The high- $T$ structure in the $a c$-plane $\left(T>T_{\mathrm{ICDW}} \sim 350 \mathrm{~K}\right)$. Arrows indicate the directions of the low- $T$ CDW displacements for the top layer of the low- $T$ bilayer structure. The blue (red) dots indicate negative (positive) out-of-plane displacements. b) The high- $T$ structure in the $b c$-plane. c) The coordinate system notation used in optical measurements. The dashed arrow $\left(q_{\mathrm{IC}}\right)$ indicates the $a c$-plane projection of the CDW wave vector $^{6}$. d) The low- $T$ bilayer structure in the $b c$-plane.

single crystals of $\mathrm{Mo}_{8} \mathrm{O}_{23}$, aiming to disentangle various order parameters contributions to the relaxation and get insight into their respective symmetries.

We found a very anisotropic transient response that develops gradually below $T_{\mathrm{IC}}$ and saturates below $T_{\mathrm{el}} \sim$ $150 \mathrm{~K}$. We observe a prominent coherent mode $(\mathrm{CM})$ arising around $T_{\mathrm{IC}}$ with an unusual non-monotonic temperature dependence of frequency and damping. A few less intense coherent modes become visible below $T_{\text {el }}$. In addition, a sub-ps exponential relaxation mode (EM) is present at all temperatures. While the appearance of EM and CM modes is a typical CDW transient reflectivity phenomenon, in the present case the decay-time of the exponential EM and the damping and frequency of the coherent $\mathrm{CM}$ demonstrate extrema around $T_{\mathrm{el}}$, far from the $\mathrm{IC}$ and $\mathrm{C}$ transitions. Overall, we find that the transient response is consistent with the presence of a (pseudo)gap in the electronic spectrum below $T_{\mathrm{IC}} \sim 350 \mathrm{~K}$ with an additional gap opening below $\sim 160 \mathrm{~K} \sim T_{\mathrm{el}}$. At the same time, the behavior of the $\mathrm{CM}$ is quite unconventional suggesting that the EM and CM are not coupled to the IC(-C) CDW order parameter only.

\section{EXPERIMENTAL AND THEORETICAL METHODS}

The samples were grown as described in the literature $21 / 35$. The single-crystal nature of the samples was confirmed by XRD. The resistivity of the samples is consistent with previous reports ${ }^{6}$ and exhibits a characteristic peak at $T_{\mathrm{el}} \sim 150 \mathrm{~K}^{21}$. The orientation of the crystal axes was determined by means of the X-ray diffraction (XRD) and the polarized optical microscopy.

The transient reflectivity studies were performed using a standard pump-probe setup using $50 \mathrm{fs}$ linearly polarized laser pulses at $800 \mathrm{~nm}$ wavelength and the $250 \mathrm{kHz}$ repetition rate as presented in detail elsewhere ${ }^{36}$. The probe-photon energy was at the laser fundamental, $\hbar \omega=1.55 \mathrm{eV}$, while the pump-photon energies were either $1.55 \mathrm{eV}$ or $3.1 \mathrm{eV}$. The transient reflectivity, $\Delta R / R$, was measured at a near-normal incidence from the $a c$ plane with the incident and reflected light propagating at $\sim 15$ degrees with respect to the crystal $b$-axis. The angle was slightly different in different runs with no noticeable effect on the results. No dependence of the signal on the pump polarization was found with the $3.1 \mathrm{eV}$ pump-photon energy. The beam diameters at the sample surface were $\sim 100 \mu \mathrm{m}$ and $\sim 50 \mu \mathrm{m}$ for the pump and the probe beams, respectively. The low fluences of the pump $F_{\mathrm{pu}}<=40 \mathrm{uJ} / \mathrm{cm}^{2}$ were verified to transiently increase lattice temperature for less than $5 \mathrm{~K}$. This agrees with an estimate based on the penetration depth of $20 \mathrm{~nm}$ from ab initid ${ }^{21}$ calculations. Samples were cleaved before experiments, but no major difference in the response from the cleaved and as-grown $a c$ crystal surfaces was observed. The temperature dependence of $\Delta R / R$ was measured with the pump fluence of $10 \mathrm{uJ} / \mathrm{cm}^{2}$ at a fixed linear probe polarization oriented approximately along the maximum of the low- $T$ anisotropic $\Delta R$. The polarization dependence of $\Delta R / R$ at selected temperatures was measured using the fluence of $\sim 40 \mathrm{uJ} / \mathrm{cm}^{2}$ with the probe fluence being $\sim 8$ times lower. In this range, the $\Delta R / R$ transients were found to scale linearly with the fluence. The characteristic changes of $\Delta R / R$ with the probe-polarization when varying temperature over the IC-C transition were always measured in a single run with a constant probe incidence angle. To measure the static reflectivity anisotropy, $R(\theta)$, the angular dependence of either the reflected $1.55-\mathrm{eV}$ probe-beam intensity or the reflected broadband supercontinuum probe-beam spectral density was measured. In the later case no in-situ reference mirror was used so the magnitude-calibrated $R(\theta)$ is available only at $1.55-\mathrm{eV}$ photon energy.

Temperature dependent Raman spectra were measured in the backscattering geometry using 10x microscope objective. The excitation laser wavelength was $632.8 \mathrm{~nm}$ with the incident laser power of a few hundred $\mu \mathrm{W}$.

\section{RESULTS}

\section{A. Raman}

In Fig. 2 we show temperature dependent Raman spectra measured in the backscattering geometry along the $b$ axis, with the analyzer parallel to the laser polarization. 

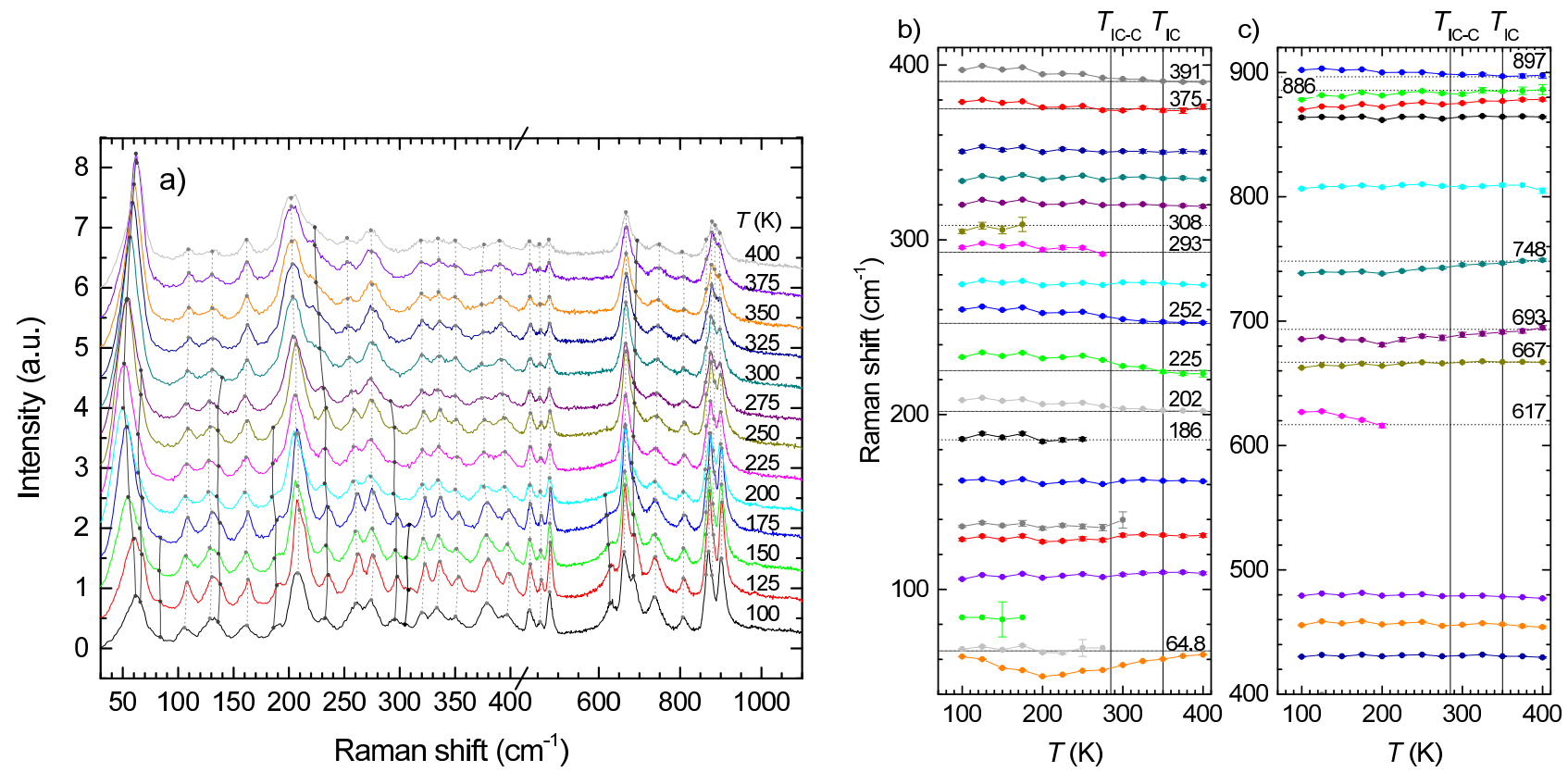

Figure 2. (a) Temperature dependent vibrational Raman spectra. The full vertical lines indicate the modes that show more significant $T$-dependent intensity. (b) and (c) Temperature dependence of the peak positions obtained by means of multiple Lorentzian-peak fits.

The orientation of the crystal in the ac plane was chosen for the largest intensity in the low frequency region.

A large number of vibrational modes is observed. No clear new modes appear at $T_{\mathrm{IC}}$. Below $T_{\mathrm{IC}-\mathrm{C}}$ a few modes (at 137, 187, 233 and $293 \mathrm{~cm}^{-1}$ ) develop to well defined peaks from broad shoulders above $T_{\mathrm{IC}-\mathrm{C}}$. Another mode at $617 \mathrm{~cm}^{-1}$ with a significant $T$-dependent frequency shift rises below $175-200 \mathrm{~K}$. The other modes show slight increase of intensity with decreasing $T$. Below $175-200 \mathrm{~K}$ some of these modes (the $478 \mathrm{~cm}^{-1}$ mode for example) show an enhanced intensity increase.

The most striking feature of the spectra is the temperature dependence of the lowest frequency $\sim 65 \mathrm{~cm}^{-1}$ mode that shows a strong softening with decreasing $T$ down to $175-200 \mathrm{~K}$ followed by hardening back to the high- $T$ frequency upon further cooling. Among the other modes some show hardening, some softening, and some also no appreciable $T$ dependence.

Most of the shifting modes show the largest frequency shifts across $T_{\mathrm{IC}-\mathrm{C}}$ with an onset of shift observable already below $T_{\text {IC }}$. A marked exception is the mode at $375 \mathrm{~cm}^{-1}$ that shows a frequency shift below $175-200 \mathrm{~K}$ only. Note that the peaks that demonstrate changes below $200 \mathrm{~K}$ are rather weak and the changes are clearly observed only below $\sim 175 \mathrm{~K}$. Thus $200 \mathrm{~K}$ is a guideline temperature corresponding to the maximal observed softening of the anomalous mode at the $\sim 65 \mathrm{~cm}^{-1}$.

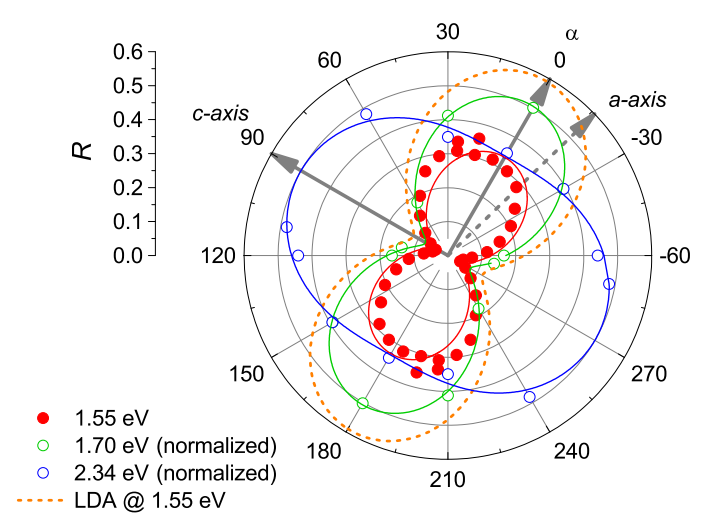

Figure 3. Anisotropy of the static reflectivity, $R$, at different photon energies. The data represented with open symbols are normalized to $R_{\max }(\theta)=0.5$ due to the lack of calibration. The continuous lines represent the fits using (Eq. 3).

\section{B. Reflectivity anisotropy}

A low monoclinic symmetry implies that one can expect a strongly anisotropic optical response so in order to analyze the transient-reflectivity polarization dependence we first need to determine the anisotropy of the static optical reflectivity at the relevant wavelengths used in the experiments. In Fig. 3 we show the static ac-plane reflectivity $R$ as a function of the polarization angle. A strong angular dependence of $R$ at $1.55 \mathrm{eV}$ and $1.70 \mathrm{eV}$ is found that becomes weaker at the highest photon energy $2.34 \mathrm{eV}$. To analyze this in more detail, we recall that 


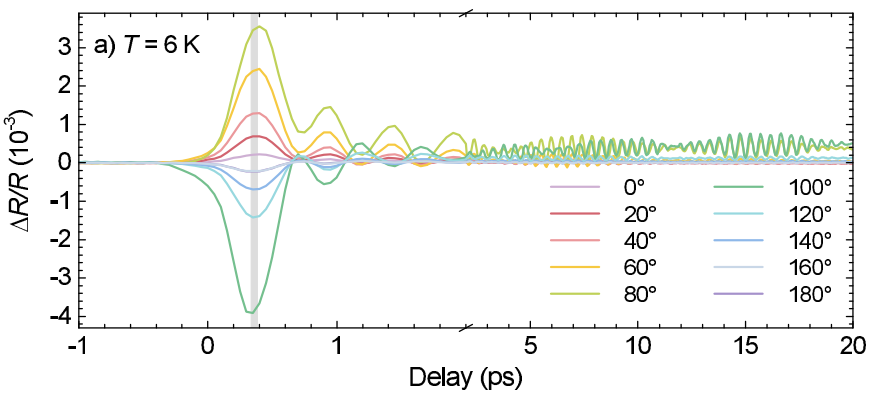

b) $T=6 \mathrm{~K}$
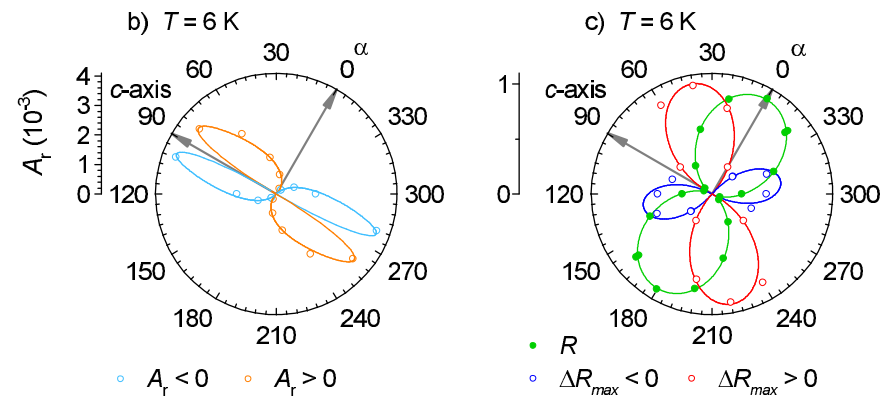
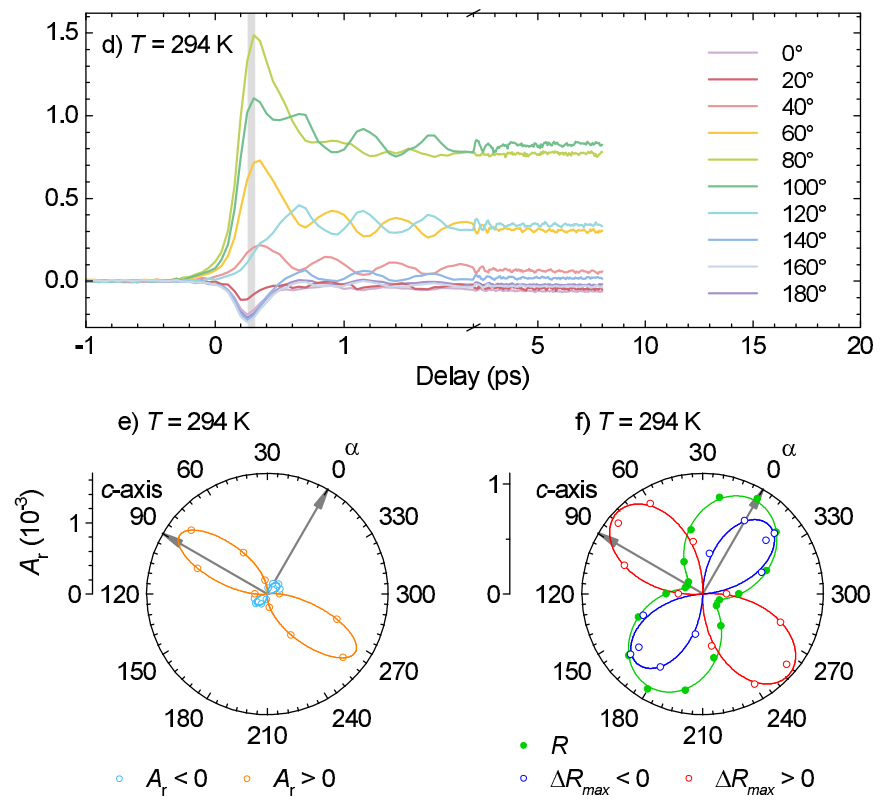

Figure 4. (a), (d) The transient reflectivity, $\Delta R / R$, as a function of the probe polarization angle at $6 \mathrm{~K}$ and $294 \mathrm{~K}$ respectively. (b), (e) Angular dependence of the transient reflectivity amplitude, $A_{\mathrm{r}}$, at $6 \mathrm{~K}$ and $294 \mathrm{~K}$ respectively. (c), (f) Comparison of the unnormalized transient reflectivity amplitude, $\Delta R_{\max }$, to the static reflectivity, $R$ (the green curve with the maximum at zero angle) at $6 \mathrm{~K}$ and $294 \mathrm{~K}$ respectively. The blue colors in (b), (c), (e) and (f) represent the negative lobes.

the monoclinic symmetry of $\mathrm{Mo}_{8} \mathrm{O}_{23}$ crystals $\frac{6[9}{\text { implies }} 37$ that the dielectric tensor has the form

$$
\hat{\epsilon}=\left(\begin{array}{ccc}
\epsilon_{\alpha \alpha} & 0 & \epsilon_{\alpha \mathrm{c}} \\
0 & \epsilon_{\mathrm{bb}} & 0 \\
\epsilon_{\alpha \mathrm{c}} & 0 & \epsilon_{\mathrm{cc}}
\end{array}\right)
$$

where $\epsilon_{i j}$ represent the complex dielectric susceptibility components in the orthogonal coordinate system, $\{\vec{\alpha}=$ $\vec{b} \times \vec{c}, \vec{b}, \vec{c}\}$, where $\vec{a}, \vec{b}$ and $\vec{c}$ correspond to the crystal-axes directions (see Fig. 1). This can in general yield complex eigenvectors of the dielectric tensor in the $a c$-plane that are wavelength-dependent. As a result the general $a c$ plane complex reflectivity is given by

$$
\hat{r}_{\mathrm{ac}}=\left(\begin{array}{cc}
r_{\alpha \alpha} & r_{\alpha \mathrm{c}} \\
r_{\alpha \mathrm{c}} & r_{c c}
\end{array}\right) .
$$

Under a polarized microscope the $\mathrm{Mo}_{8} \mathrm{O}_{23}$ single crystals show good cross-polarized extinction with the incident polarization along the $\alpha$ and $c$ axes (within the experimental error of $\sim \pm 5^{\circ}$ ) implying that $\left|r_{\alpha c}\right| \ll\left|r_{c c}\right|<$ $\left|r_{\alpha \alpha}\right|$. The observed dependence can therefore be fit with

$$
R(\theta)=C_{1}+C_{2} \cos (2 \theta)
$$

where $\theta$ is the angle relative to the $\alpha$ axis.

In Fig. 3 we also show for comparison the reflectivity anisotropy calculated by means of the $\mathrm{DFT}^{21}$ for $\mathrm{Mo}_{8} \mathrm{O}_{23}$ in the high-temperature structure. Overall, the calculations yield a similar anisotropy as the observed one, but the magnitude of the calculated reflectivity is approximately a factor of 2 larger than the measured values.
Overall we believe that discrepancies are within the accuracy of DFT (LDA) calculations. For the photon energies other than $1.55 \mathrm{eV}$ the DFT (LDA) calculations also demonstrate qualitatively the same anisotropies as experimental ones.

\section{Transient reflectivity: anisotropy}

The low $T$ transient reflectivity, $\Delta R / R$ shown in Fig. 4 (a) is dominated by the coherent oscillatory response. A clear beating of the oscillatory responses indicates the presence of at least two modes.

The angular dependence of the $\Delta R / R$ amplitude, $A_{\mathrm{r}}$, shown in Fig 4 (b) and (e) shows a peculiar angular dependence. The unnormalized $\Delta R_{\max }$ shows a phaseshifted angular dependence of the similar form (Eq. 3), $\Delta R_{\max } \sim 1+C_{3} \cos \left(2\left[\theta-\theta_{0}\right]\right)$, shown in Fig. 4 (c) and (e). The peculiar shape is therefore a result of the angular phase shift, $\theta_{0} \sim 40^{\circ}$ at $T=6 \mathrm{~K}$, of $\Delta R_{\max }(\theta)$ with respect to $R(\theta)$,

$$
A_{\mathrm{r}} \sim \frac{\Delta R_{\max }(\theta)}{R(\theta)} \sim \frac{1+C_{3} \cos \left(2\left[\theta-\theta_{0}\right]\right)}{1+\frac{C_{2}}{C_{1}} \cos (2 \theta)}
$$

The $A_{\mathrm{r}}$ angular dependence shows a handedness that was different in different crystals, but was never observed to change upon temperature cycling. This is consistent with the symmetries of both the high- $T(P 2 / c)$ and the low- $T(P c)$ crystal space group that allow a pseudovector 

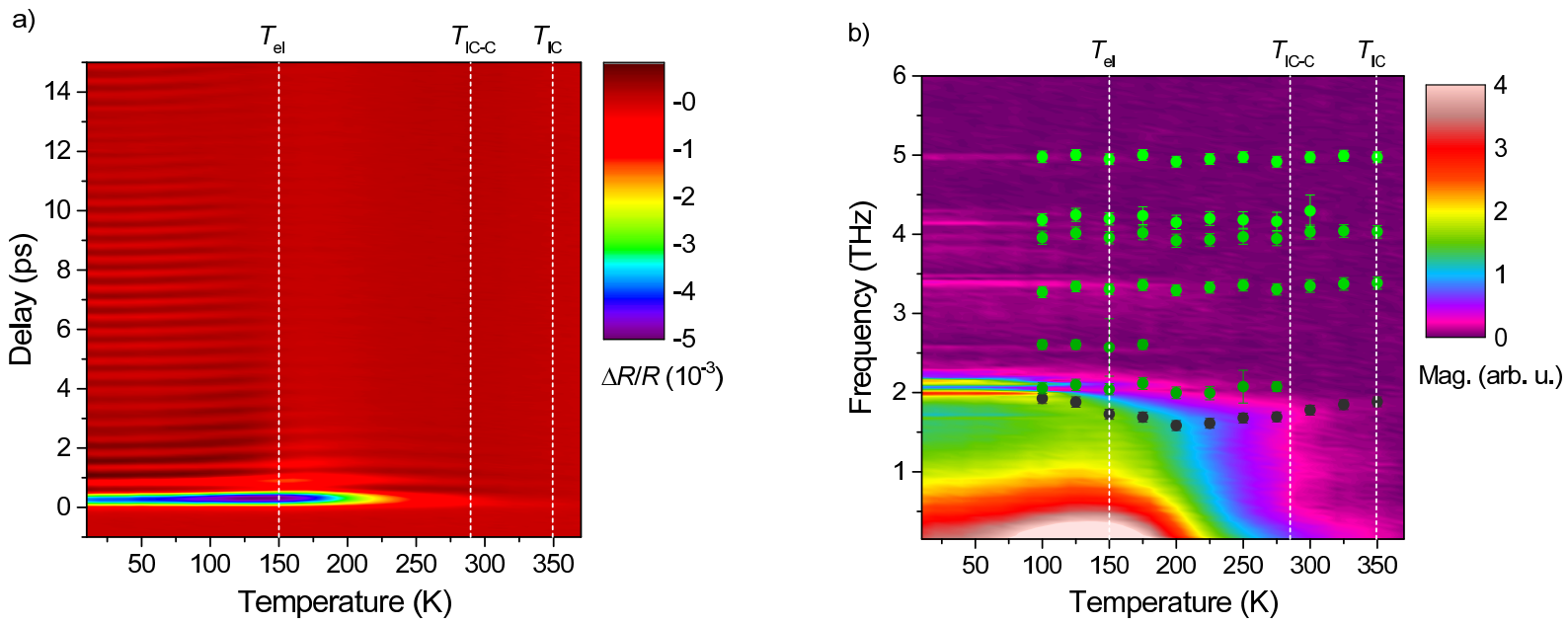

Figure 5. (a) Temperature dependence of the transient reflectivity measured at the $10 \mu \mathrm{J} / \mathrm{cm}^{2}$ pump fluence and $\theta \sim 40^{\circ}$. (b) The Fourier transform of the data presented in (a). Full symbols correspond to the frequencies of the low frequency Raman peaks. The vertical dashed lines indicate the transition temperatures: $T_{\mathrm{IC}} \sim 350 \mathrm{~K}$ and $T_{\mathrm{IC}-\mathrm{C}}=285 \mathrm{~K}$ and $T_{\mathrm{el}}$.
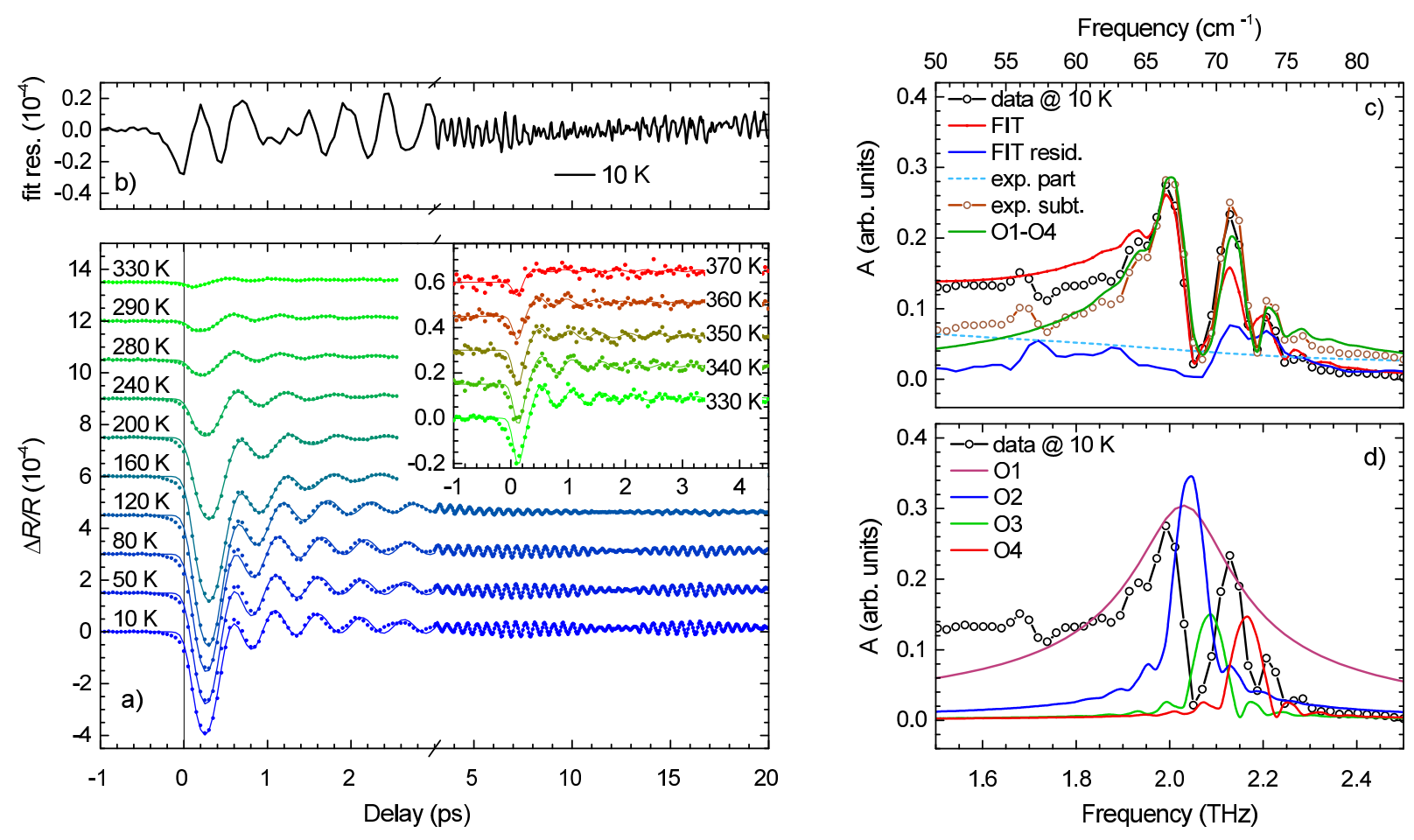

Figure 6. (a) DCE model (Eq. 55 fits (lines) to the transient-reflectivity data (circles). The high- $T$ data are shown in the inset. The full decomposition of the raw data at $T=10 \mathrm{~K}$ into the components is shown in Appendix. (b) The fit residuum at $T=10 \mathrm{~K}$ reveals the weaker high frequency modes. (c) The Fourier transform amplitude of the transients and fit components at $T=10 \mathrm{~K}$. (d) The $T=10 \mathrm{~K}$ Fourier transform amplitude of the four fit coherent modes compared to the data. The lineshapes of the two narrowest coherent modes are due to the finite scan-length. The side lobes of all the modes are also a consequence of the finite scan-length.

along the $b$-axis. Different handedness in different crystals can be related to different orientation of the particular crystal pseudovector relative to the light propagation direction.

\section{Transient reflectivity: temperature dependence}

The temperature dependence of the transient reflectivity was measured at $\theta \sim 40^{\circ}$ where the low- $T \Delta R_{\max }$ shows the maximum. The transient reflectivity is shown in Fig. 5(a). One can see clear coherent oscillations that 
appear soon after cooling below $T_{\mathrm{IC}}$. Also on cooling, the amplitude of the transient reflectivity on short timescales $(<1 \mathrm{ps})$ begins to increase. Similar results were reported for other CDW compounds ${ }^{23139}$, including $\mathrm{Mo}_{4} \mathrm{O}_{11}$, another member of the $\mathrm{MoO}_{3-x}$ family 4 .

The corresponding Fourier transformation of the data is shown in [Fig. 5(b)]. One can see several modes with the most intensive group centered around $2 \mathrm{THz}$. These modes are strongly damped above $250 \mathrm{~K}$. The higher frequency modes are rather weak with narrow linewidths and are clearly observed only at low $T$.

To analyze the signal we fit the data using the displacive coherent excitation model ${ }^{41}$ (DCE) where the transient reflectivity is given by

$$
\begin{aligned}
\frac{\Delta R}{R}= & \left(A_{\mathrm{displ}}-\sum A_{\mathrm{O} i}\right) \int_{0}^{\infty} G(t-u) e^{-u / \tau_{\mathrm{displ}}} d u \\
& +\sum A_{\mathrm{O} i} \int_{0}^{\infty} G(t-u) e^{-\gamma_{i} u}\left[\cos \left(\Omega_{i} u\right)\right. \\
& \left.\quad-\beta_{i} \sin \left(\Omega_{i} u\right)\right] d u \\
& +\sum_{j \in\{1,2\}} A_{\mathrm{e} j} \int_{0}^{\infty} G(t-u) e^{-u / \tau_{j}} d u,
\end{aligned}
$$

where $\beta_{i}=\left(1 / \tau_{\text {displ }}-\gamma_{i}\right) / \Omega_{i}$ and $G(t)=$ $\sqrt{2 / \pi} \tau_{\mathrm{p}} \exp \left(-2 t^{2} / \tau_{\mathrm{p}}^{2}\right)$ with $\tau_{\mathrm{p}}$ being the effective pumpprobe pulse cross-correlation width. In the DCE model the coherent modes are driven with an exponentially relaxing displacive mode (ERDM) with the relaxation time $\tau_{\text {displ }}$ and the amplitude, $A_{\text {displ }} . A_{\mathrm{O} i}, \Omega_{i}, \gamma_{i}$ are the oscillating modes amplitudes, frequencies and damping factors, respectively, while $A_{\mathrm{e} j}$ and $\tau_{j}$ are the amplitudes and relaxation times of two additional exponentially relaxing modes that are necessary to fit the data: component e1 that describes the picosecond-scale relaxation and component $\mathrm{e} 2$ which accounts for the long-lived ( $>1 \mathrm{~ns}$ ) offset (see Appendix for the decomposition of the raw data at $10 \mathrm{~K}$ into the components). To reduce the number of parameters we ignore the weak and featureless high frequency modes and focus on the strongest group of modes around $\sim 2 \mathrm{THz}$.

In Fig. 6(a) we show the resulting fits along the measured data. Overall, the fit is good, as seen also from the Fourier transform shown in Fig. 6(c), and small fit residuals, Fig. 6(b). For this high quality of the fit, in particular to reproduce the double-peak structure around $\sim 2 \mathrm{THz}$, four oscillatory modes labeled $\mathrm{O} 1-\mathrm{O} 4$ in $\mathrm{Ta}-$ ble I are necessary.

Let us describe the temperature dependence of those oscillatory modes. Fig. 7 shows the temperature dependence of the fitted frequencies and the amplitudes. On cooling the most prominent mode $\mathrm{O} 1$ appears just above $T_{\text {IC }} \sim 350 \mathrm{~K}$ with a strong increase of the amplitude below $\sim 300 \mathrm{~K}$. The mode frequency and rather large damping (linewidth) strongly depend on temperature. Interesting non-monotonic behavior is observed: upon decreasing $T$ the mode first softens from $\Omega_{1} / 2 \pi \sim 2.1 \mathrm{THz}$ to the minimum frequency of $\sim 1.7 \mathrm{THz}$ at $T_{\mathrm{m}} \sim 200 \mathrm{~K}$

\begin{tabular}{c|c|c|c} 
& $\omega / 2 \pi(\mathrm{THz})$ & $\gamma / 2 \pi(\mathrm{THz})$ & $A$ (arb. u.) \\
\hline O1 & 2.02 & 0.12 & 28.4 \\
O2 & 2.04 & 0.007 & -5.7 \\
O3 & 2.09 & $<0.007$ & -1.5 \\
O4 & 2.17 & $<0.007$ & -1.4
\end{tabular}

Table I. The four strongest coherent mode fit parameters at $T=10 \mathrm{~K}$.

and then hardens with further cooling to $2.02 \mathrm{THz}$. The mode damping shows a broad maximum $\left(\gamma_{\max } / 2 \pi \sim\right.$ $0.24 \mathrm{THz}$ ) spread between $\sim 200 \mathrm{~K}$ and $\sim 100 \mathrm{~K}$.

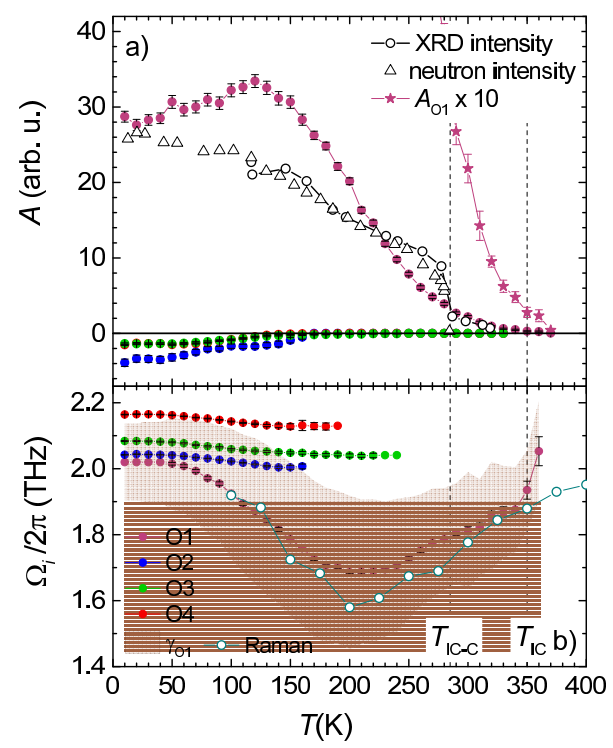

Figure 7. (a) Temperature dependence of the coherent mode amplitudes compared to the structural order parameters from ${ }^{6}$. Temperature dependence of the coherent mode frequencies (b). The open symbols correspond to the Raman scattering $65-\mathrm{cm}^{-1}$ mode frequency (mode RO1). The linewidth of the dominant coherent mode $\mathrm{O} 1$ is shown by the shaded area.

The three additional fitted modes $\mathrm{O} 2$ - O4 become observable in the $250-170 \mathrm{~K}$ range, depending on the mode, and show smaller damping (see Table I) and weaker $T$ dependence (Fig. 7). The minor higher frequency modes (above $2.5 \mathrm{THz}$ ) also appear at different temperatures below $170-200 \mathrm{~K}$ with a general trend of the higher frequency modes appearing at lower $T$.

In Fig. 8 we show the amplitudes of two main nonoscillatory components, the ERDM $\left(A_{\text {displ }}\right)$ and the picosecond component e1 $\left(A_{e 1}\right)$ as well as the corresponding relaxation times. The ERDM is always present and its amplitude dominates the signal in the full temperature range. Its amplitude and the relaxation time show a smooth weak evolution across $T_{\mathrm{IC}}$ and the lock-in transition at $T_{\mathrm{IC}-\mathrm{C}}$. Below $\sim 250 \mathrm{~K}$ weaker picosecond component e1 appears. Its amplitude changes sign close to $150 \mathrm{~K}$. Close to this temperature both relaxation times 


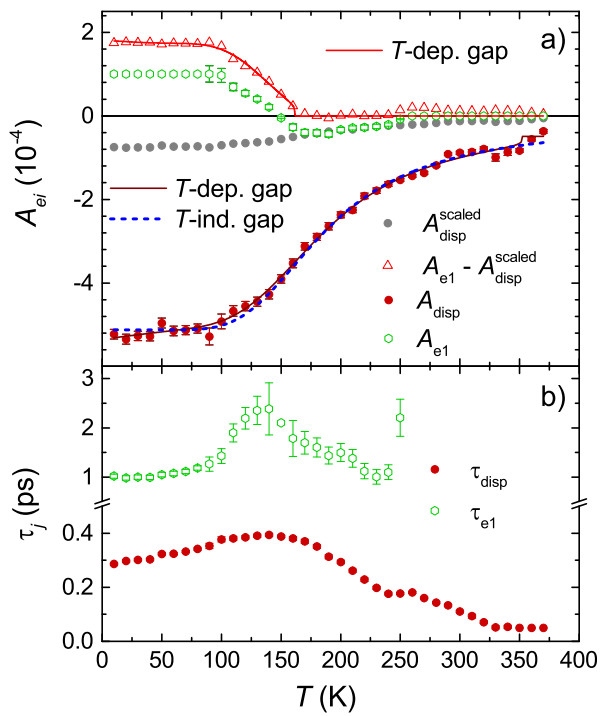

Figure 8. Temperature dependence of the two fast nonoscillating component parameters: (a) the amplitudes and (b) the corresponding decay times. The lines in (a) show the bottleneck-model fits with the temperature-independent gap (Eq. 8) (dashed line) and the temperature-dependent BCS gap (Eq. 9) (full lines). See text for more details.

are maximal.

\section{E. Transient reflectivity: T-dependent anisotropy and evidence of twinning.}

Due to the strong static reflectivity anisotropy it is instrumental to measure and analyze the $T$-dependence of the unnormalized $\Delta R$ anisotropy. In Fig. 9 we show the angular dependence of the amplitude for the two most prominent DECP components at a few characteristic temperatures. The angular-dependent DECP amplitudes at any given $T$ were obtained by means of a global fit of Eq. (5) to $\Delta R$ measured at different polarization angles. In the global fit only the amplitudes were allowed to vary with the polarization angle whereas the frequencies, dampings and relaxation times were kept angle independent. Similar as the total $\Delta R_{\max }$ [see Fig. 4 c)] the angular dependence of the individual DECP amplitudes can be well described with a phase shifted harmonic function with an offset:

$$
A(\theta) \propto 1+C_{3} \cos \left[2\left(\theta-\theta_{0}\right)\right] .
$$

At low $T$ the angular phase shift, $\theta_{0} \sim 40^{\circ}$, is similar for all DECP components. With increasing $T$, however the oscillatory components show $T$-independent $\theta_{0} \sim 40^{\circ}$, while the ERDM shows a gradual rotation towards $\theta_{0} \sim$ $90^{\circ}$.

We also found that the transient-reflectivity angular dependence in growth-mirror-twins is mirrored across the

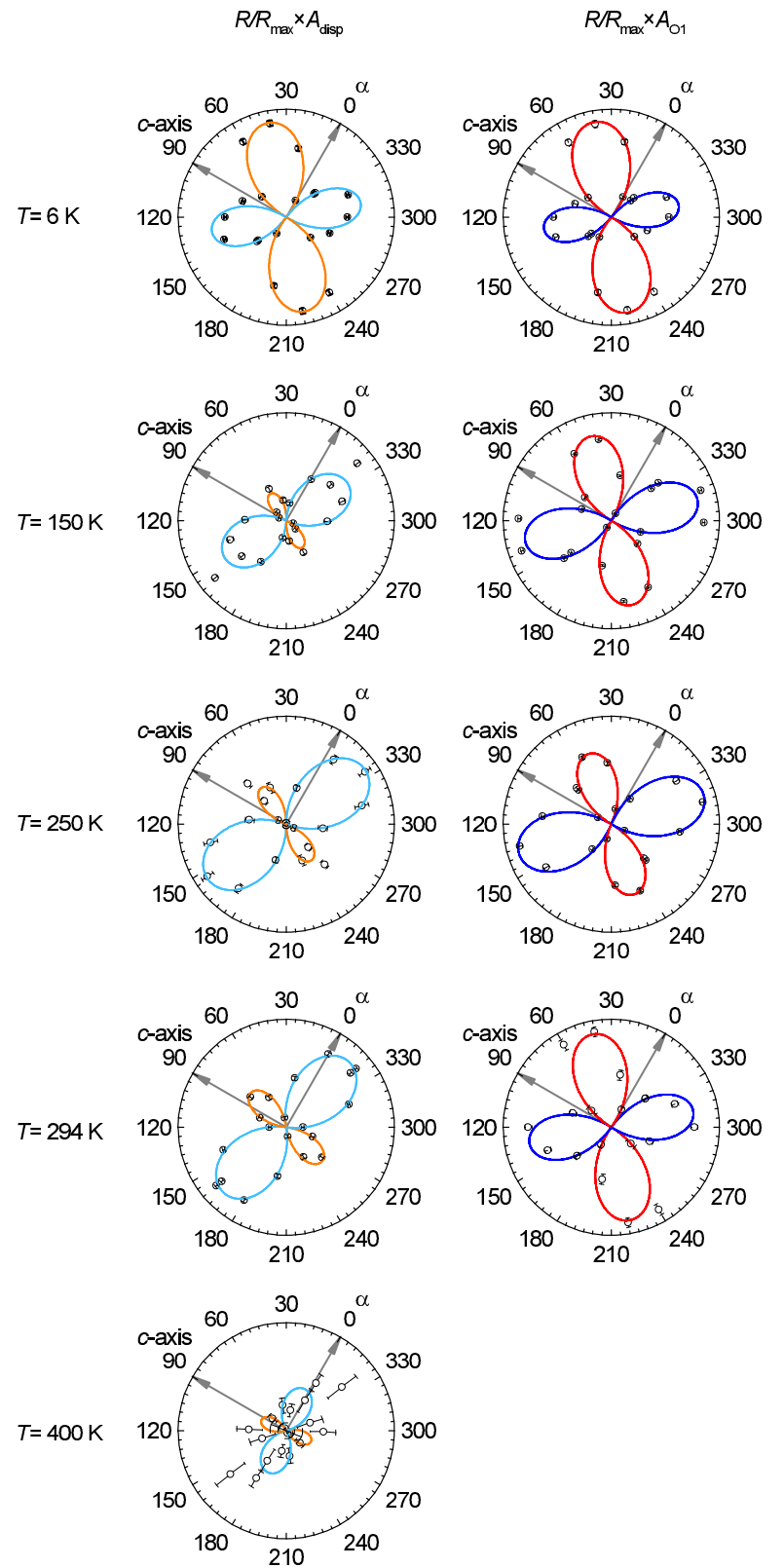

Figure 9. The main DECP component amplitudes as a function probe polarization angle at different $T$. The left and right column correspond to the ERDM and oscillatory mode O1, respectively. The solid lines are fits using Eq. (6) where the red colors correspond to the positive lobes, and the blue colors to the negative lobes.

twin mirror plane. The observed angle between the optically determined $c$-axes of the mirror-twins is $\sim 25^{\circ}$ which is close to $32.5^{\circ}$, the angle expected for the very common $\{100\}$ monoclinic twins. The difference can be attributed to the $\sim \pm 5^{\circ}$ error of the experimentally determined reflectivity tensor (Eq. 2) orientation relative to the crystal axes. 


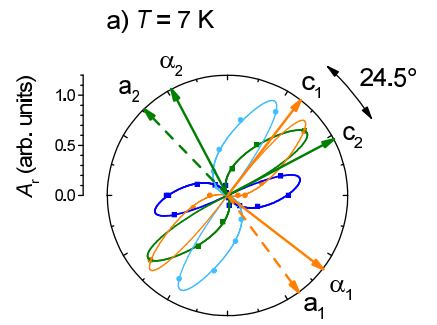

b) $T=294 \mathrm{~K}$

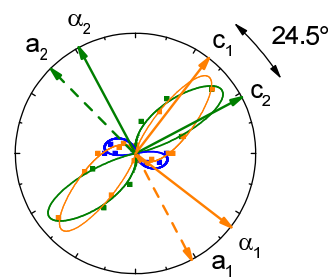

c) $T=7 \mathrm{~K}$

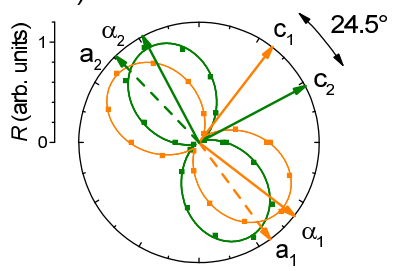

Figure 10. Comparison of the transient reflectivity anisotropy in growth twin crystals. (a) and (b) transient reflectivity amplitude at two different temperatures: $T=7 \mathrm{~K}$ and $T=294 \mathrm{~K}$ respectively. (c) Reflectivity at $T=7 \mathrm{~K}$. The data are normalized for the sake of comparison. The orientation of the axes was inferred from the static reflectivity. The blue colors indicate the negative lobes.

\section{DISCUSSION}

\section{A. Symmetry considerations}

Similar as the Raman tensor the optical transient dielectric tensor $\Delta \hat{\epsilon}$ can be reduced according to the irreducible representations of the crystal point group. The high- $T$ point group $\left(C_{2 \mathrm{~h}}\right)$ has center of inversion where $\mathrm{A}_{\mathrm{g}}$ and $\mathrm{B}_{\mathrm{g}}$-symmetry modes can couple to $\Delta \hat{\epsilon}$. The $\mathrm{B}_{\mathrm{g}}$-symmetry tensor ${ }^{42}$ has zero $a c$-plane components and does not apply to our experimental geometry, while the ac-plane part of the $\mathrm{A}_{\mathrm{g}}$-symmetry tensor has the lowest possible symmetry for a symmetric tensor:

$$
\Delta \hat{\epsilon}_{\mathrm{ac}}^{\mathrm{Ag}}=\left(\begin{array}{cc}
\Delta \epsilon_{\alpha \alpha} & \Delta \epsilon_{\mathrm{d}} \\
\Delta \epsilon_{\mathrm{d}} & \Delta \epsilon_{\mathrm{cc}}
\end{array}\right) .
$$

Upon loosing center of inversion in the low- $T$ point group $\left(\mathrm{C}_{s}\right)$, the $\mathrm{A}_{\mathrm{g}}$ and $\mathrm{B}_{\mathrm{g}}$ representations of $C_{2 h}$ reduce to A' and A" representations, respectively, without changes in the allowed Raman tensor components. The A"symmetry tensor therefore also does not apply to our experimental geometry so all the observed modes correspond to the $\mathrm{A}^{\prime}$ representation. In addition, $\mathrm{A}_{\mathrm{u}}$ and $\mathrm{B}_{\mathrm{u}}$ representations also reduce to $\mathrm{A}^{\prime}$ and $\mathrm{A}$ " representations, respectively, enabling coupling of the polar modes to $\Delta \hat{\epsilon}$ below $T_{\mathrm{IC}}$. The A' modes have allowed dipole moment lying in the ac-plane.

When measuring the angular dependence of the transient reflectivity the transient dielectric tensor shape (Eq. 7) would result in maximum/minimum at an arbitrary angle depending on the relative values of the three independent components. The gradual rotation of the ERDM with $T$ can therefore be assigned to different $T$-dependences of the $\Delta \hat{\epsilon}_{\text {ac }}$ components. The different $T$-dependencies indicate, however, that the nature of the involved electronic states changes with $T$. This is further corroborated with an appearance of the additional picosecond component and increase of intensities of Raman modes below $175-200 \mathrm{~K}$.

\section{B. Electronic relaxation and the non-oscillatory components}

To investigate the single-particle excitations, it is interesting to consider the data in Fig. 8 in more detail. As discussed above, the two non-oscillatory components ERDM and e1, associated with the overdamped electronic relaxation processes, show an unusual temperature evolution around $T_{\mathrm{el}}$ where the resistivity hump occurs. On the other hand, they show no conventional critical behavior of their relaxation time, that could be expected at the CDW transitions at $T_{\mathrm{IC}}$ or $T_{\mathrm{IC}-\mathrm{C}}$. In contrast, an increase of the relaxation times, in particular $\tau_{\mathrm{e} 1}$ is quite prominent close to $T_{\mathrm{el}}$, which could indicate a critical point that is not related to the established transitions at higher temperatures.

A picosecond time scale in electronic relaxation and an increase of the amplitude of the corresponding electronic response with cooling is expected in gapped systems. From the electronic structure calculations ${ }^{14 \mid 21}$, we expect a single-particle density-of-states characteristic of a semi-metal or a narrow band semiconductor $(2 \Delta \sim$ $0.1 \mathrm{eV})$. Indeed, one can understand the $T$-dependencies of the amplitudes $A_{\mathrm{displ}}$ and $A_{\mathrm{e} 1}$ in terms of a gapped behavior in single-particle excitations. To look into this quantitatively, we apply a Rothwarf-Taylor type bottleneck model 43/44, that describes electronic relaxation via phonons in systems with a $T$-independent gap of a magnitude $2 \Delta$. Within this model the change of reflectivity is

$$
\frac{\Delta R}{R} \propto n_{\mathrm{ph}} \propto\left[1+\gamma \exp \left(-\frac{\Delta}{k_{B} T}\right)\right]^{-1},
$$

where $n_{\mathrm{ph}}$ corresponds to the photoexcited single particle excitations density. The parameter $\gamma=\frac{2 \nu}{N(0) \hbar \Omega_{C}}$ is the ratio between the number of the involved bosonic and electronic degrees of freedom. Here $\hbar \Omega_{C} \sim 2 \Delta$ corresponds to the characteristic bottleneck-phonon frequency, $\nu$ to the number of the involved phonon modes and $N(0)$ to the number of the involved electronic states.

The temperature dependence of $A_{\text {displ }}$ is fitted well with this formula, as shown in Fig 8 (a). The fit yields $2 \Delta=137 \pm 10 \mathrm{meV}$ and $\gamma \sim 60$. Based on the LDA it is reasonable to take $N(0) \sim 10 \mathrm{eV}^{-1}$ [21, which yields $\nu \sim 30$. The number is similar to $\nu=10-20$, obtained in the cuprates 44,45 .

We also fit $A_{\text {displ }}$ within a temperature-dependent BCS-type gap bottleneck model $44 \Delta_{\mathrm{BCS}}(T)$ with $T_{\mathrm{c}}=$ 
$T_{\mathrm{IC}} \sim 350 \mathrm{~K}$ to see whether one could interpret the results in terms of the opening of a CDW gap $\stackrel{46}{ }$ In this case

$$
\begin{aligned}
\frac{\Delta R}{R} \propto & {\left[\Delta_{\mathrm{BCS}}(T)+\frac{k_{B} T}{2}\right]^{-1} } \\
& \times\left\{1+\gamma \sqrt{\frac{k_{B} T}{\Delta_{\mathrm{BCS}}(T)}} \exp \left[-\frac{\Delta_{\mathrm{BCS}}(T)}{k_{B} T}\right]\right\}^{-1},
\end{aligned}
$$

yields similar parameter values: $2 \Delta_{\mathrm{BCS}}(0)=112 \pm$ $10 \mathrm{meV}$ and $\gamma \sim 45$. The BCS type temperature dependent gap function describes the amplitude, $A_{\text {disp }}$, equally well as the temperature independent one, so the temperature dependence of the gap at high temperatures cannot be discerned from these fits alone.

On the other hand, the absence of a relaxation time divergence at $T_{\mathrm{IC}}$ and $T_{\mathrm{IC}-\mathrm{C}}$ strongly suggests that there is no rapid BCS-like change in the DOS at the IC and IC-C transitions, so a T-independent gap is more appropriate in this case.

Within the same approach, we also considered the temperature dependence of the picosecond component amplitude, $A_{\mathrm{e} 1}$. In order to analyze this we subtracted from $A_{e 1}$ a part proportional to $A_{\text {displ }}$ to remove the negative high-temperature tail shown in Fig. 8 a ${ }^{47}$ Both the temperature independent and the BCS-type model again can fit the temperature dependence of $A_{e 1}$ quite well. However, the temperature-independent-gap fit yields an unphysical value of $\gamma \sim 10000$, which suggests that a temperature independent gap is not appropriate in this case. Rather, assuming a temperature-dependent BCStype gap yields $2 \Delta_{\mathrm{BCS}}(0)=85 \pm 10 \mathrm{meV}, \gamma \sim 26$ and $T_{\mathrm{c} 1}=160 \pm 5 \mathrm{~K}$. Importantly, the peak in $\tau_{\mathrm{e} 1}$ observed just below $T_{\mathrm{c} 1}$ is consistent with opening of an additional low- $T$ gap.

The relatively decoupled transient reflectivity dynamics of the two exponential components despite similar gap magnitudes suggests that the high- $T$ and low- $T$ gaps are separated in $k$-space and the momentum scattering (impurity and acoustic phonon) between the relevant electronic states must be weak. These data suggest that the band structure contains multiple gaps, in different regions of the Brillouin zone.

\section{Oscillating modes}

We now turn to a closer discussion of the oscillatory modes. The dominant observed coherent mode O1 appears below $T=360 \mathrm{~K}$ and shows an unusual behavior of the frequency and damping. It directly corresponds to the lowest frequency Raman mode (RO1). In Raman spectra (RS) the mode does not show a significant intensity change across the IC and IC-C transitions so it is not a folded mode or the IC-transition soft mode. The weak $T$-dependence of the mode RS intensity indicates that the main contribution to the strong O1-mode coherent amplitude $T$-dependence is due to the $T$-dependence of the displacive component drive amplitude. The minor difference between the $T$-dependence of $A_{\mathrm{O} 1}$ and $A_{\text {disp }}$ can be attributed to the temperature dependence of $\tau_{\text {disp }} 41$ and/or slight $T$-dependence of the mode coupling to the reflectivity. Moreover, component e1 in principle also contributes to the displacive drive. This is not explicitly included in the model (5), but, since $A_{\mathrm{O} 1}$ and $A_{\text {disp }}$ are independent fit parameters $A_{\mathrm{O} 1}$ would pick such contribution.

The strong softening of $\sim 0.2 \mathrm{THz}$ observed in a relatively narrow $T$ range around the nominal $T_{\mathrm{IC}} \sim 350 \mathrm{~K}$ is absent in RS. This indicates that the coherent oscillations observed at $T=360 \mathrm{~K}$ most likely correspond to weaker mode O3 that is obscured by mode O1 in the $350-250 \mathrm{~K} T$-range and is detectable only at lower temperatures. Both $\mathrm{O} 1$ and RO1 show strong softening upon cooling to $T_{\mathrm{m}} \sim 200 \mathrm{~K}$ and then harden back with further cooling. The RO1 minimum frequency appears slightly lower than the $\mathrm{O} 1$ minimum frequency.

The $T$-dependence of $\Omega_{\mathrm{O} 1}$ is anomalous and clearly unrelated to the ordering either at $T_{\mathrm{IC}}$ of $T_{\mathrm{IC}-\mathrm{C}}$. The modeO1 maximum damping is near $T_{\mathrm{m}}$, rather than near the either of the transition temperatures 48 . Since it is not a folded mode it cannot be explained within the simple CDW-like folded-phonon-mode scenario ${ }^{49}$ related to the IC transition. Its behavior would be the most similar to the folded CDW mode behavior in the case of a strong ("non-adiabatic") coupling between a phonon and a CDW electronic mode (EM) $\stackrel{[99}{\leftrightarrows}$ When the temperature dependent relaxation rate of the EM crosses the phonon frequency, their mixing causes a phonon softening anomaly and strong critical broadening with increasing temperature. But in our case no critical EM is observed and the broadening near $T_{\mathrm{IC}}$ is much smaller than in the case of a CDW mode.

The other coherent modes $\mathrm{O} 2-\mathrm{O} 4$, and the remaining weak modes (that were not fit), obtain a considerable intensity well below $T_{\mathrm{IC}}$ an $T_{\mathrm{IC}-\mathrm{C}}$ but show a much weaker frequency $T$-dependence. The modes are not completely resolved in RS due to the worse frequency resolution and appear as weaker features in RS. Similar to mode RO1 they are present ${ }^{50}$ above $T_{\text {IC }}$ in RS and show no abrupt intensity/shift change at $T_{\mathrm{IC}}$ and $T_{\mathrm{IC}-\mathrm{C}}$. The strong $T$-dependences of their amplitudes in the coherent response are therefore attributed as for mode $\mathrm{O} 1$ to the $T$-dependence of the displacive-component-drive amplitude.

The coherent modes O2-O4 frequency $T$-dependencies are small and resemble the shape of the $\Omega_{\mathrm{O} 1}$ vs $T$ dependence below $\sim 150 \mathrm{~K}$, presumably due to repulsion resulting from their weak coupling 51 to mode O1. Their smaller frequency shift indicates that they do not play an active role in the electronic changes observed below $\sim 200 \mathrm{~K}$.

In addition to the coherent response RS also show sensitivity to the electronic changes observed below $\sim 200 \mathrm{~K}$. Some modes show a clear increase of intensity. There are also some additional frequency shifts below $175-200 \mathrm{~K}$ 
that are the most notable for the $617 \mathrm{~cm}^{-1}$ and $375 \mathrm{~cm}^{-1}$ modes.

The IC and IC-C transitions appear more clearly in the behavior of some higher frequency Raman modes. These modes show small frequency shifts (either hardening or softening) that can be well correlated with the $\mathrm{IC} / \mathrm{C}$ structural order parameter shown in Fig. 7 a). (see for example the $525 \mathrm{~cm}^{-1}$ and $748 \mathrm{~cm}^{-1}$ modes in Fig. 2 . Some of these modes show also additional frequency anomalies below $\sim 200 \mathrm{~K}$ (see for example the $391 \mathrm{~cm}^{-1}$ and $639 \mathrm{~cm}^{-1}$ modes).

\section{The enigmatic state at low $T$}

Based on the discussion above, the transient reflectivity across the $\mathrm{IC}$ and $\mathrm{IC}-\mathrm{C}$ transitions of $\mathrm{Mo}_{8} \mathrm{O}_{23}$ does not behave as a typical CDW system. The anomalous behavior in $\mathrm{Mo}_{8} \mathrm{O}_{23}$ at low $T$ is apparently driven by something seemingly quite unrelated to either of the $350 \mathrm{~K}$ (IC) or $285 \mathrm{~K}(\mathrm{C})$ transitions. It is particularly interesting that the electronic relaxation shows clear evidence of a gap opening upon cooling below $T \sim 150 \mathrm{~K}$, even showing signs of a divergence of the lifetime near this temperature, while at the same time, the resistivity starts to drop. A simple explanation is that the carriers that dominate the transport properties are separated in $k$-space from the gapped excitations that are observed in the timeresolved spectroscopy. However, the anomalies in both channels seemingly capture the temperature evolution of the electronic structure beyond the reported transitions.

Although we observe a gap-related bottleneck below $T_{\text {IC }}$, our data suggest that the low-energy electronic band structure evolves rather smoothly across the IC(-C) transitions without a large immediate DOS loss at $T_{\mathrm{IC}}$ and $T_{\text {IC-C. }}$. The anomalous large softening of mode $\mathrm{O} 1$ below $350 \mathrm{~K}$ and the appearance of the additional Raman mode below $175-200 \mathrm{~K}$ might be explained by the existence of an additional hidden electronic ordering transition at low temperatures.

On the other hand, the $\mathrm{IC} / \mathrm{C}$ structural order parameter $^{6}$ (in Fig. 7 a) shows a large continuous increase below $\sim 280 \mathrm{~K}$ down to $\sim 100 \mathrm{~K}$. The continuously increasing structural order parameter together with a possible chemical potential shift could lead to a Lifshitz transition $\stackrel{52 / 53}{5}$ and/or an additional gap opening below $175-200 \mathrm{~K}$. The observed low- $T$ gap opening in the transient response and changes in transport ${ }^{21}$ would be compatible also with such scenario. However, the large mode O1/RO1 softening and the changes in the Raman spectra below $175-200 \mathrm{~K}$ cannot be directly linked to the structural order parameter induced changes. The mode O1/RO1 softening could be associated with an incipient Fermi-surface driven instability that is eventually suppressed by the competing IC/C-order-induced low-energy DOS suppression while the absence of the $617 \mathrm{~cm}^{-1}$ above $175-200 \mathrm{~K}$ would be attributed to a stronger decrease of the resonant Raman cross-section in comparison to the other modes.

The incipient transition scenario is also favored by the discrepancy of the maximum softening temperature, $T_{\mathrm{m}} \sim 200 \mathrm{~K}$, that is somewhat higher than the low- $T$ gap opening temperature $T_{\mathrm{c} 1} \sim 160 \mathrm{~K} \sim T_{\mathrm{el}}$.

Another possible explanation for the difference between $T_{c 1}$ and $T_{\mathrm{m}}$ would be the presence of a rather broad fluctuation region where different couplings of different probes result in the apparently different transition temperatures as was observed for the pseudogap in the cuprates.

\section{SUMMARY AND CONCLUSIONS}

The transient-reflectivity and vibrational Raman spectra in $\mathrm{Mo}_{8} \mathrm{O}_{23}$ single crystals reveal significant details on the evolution of the electronic structure and collective mode behavior associated with different ordering phenomena in this unusual material.

Below the incommensurate transition at $T \sim 350 \mathrm{~K}$ the temperature evolution of the fastest sub-picosecond transient relaxation component indicates a bottleneck due to the presence of a temperature independent (pseudo- ) gap with a magnitude $2 \Delta=140 \mathrm{meV}$. The absence of any significant singularity in any of the transient reflectivity components at either the incommensurate transition temperature $T_{\mathrm{IC}} \sim 350 \mathrm{~K}$, or the incommensuratecommensurate transition at $T_{\mathrm{IC}-\mathrm{C}}=285 \mathrm{~K}$ suggests only a minor modification of the low-energy DOS takes place at these transitions. Moreover, no additional Raman active vibrational modes appear at either of the transitions that could be assigned to a soft mode, so the behavior at the IC and IC-C transitions is highly unconventional.

Even more enigmatic is the picosecond relaxation component dynamics that suggests an opening of a previously unknown temperature-dependent gap $2 \Delta(0) \sim 90 \mathrm{meV}$ around $T=160 \mathrm{~K}$, that is apparently unrelated to the previously observed transitions. ${ }^{6}$ The most prominent coherent mode and its Raman counterpart at $\sim 2 \mathrm{THz}$ show anomalous $\sim 20 \%$ softening around $T_{\mathrm{m}} \sim 200 \mathrm{~K}$, with accompanying anomalies of some of the higher frequency Raman modes. Altogether, the observed anomalous behavior points towards the presence of a previously unreported hidden electronic transition below $T_{\mathrm{c} 1} \sim 160 \mathrm{~K}$.

The Brillouin-zone-center lattice dynamics anomalies observed well above $T_{\mathrm{c} 1} \sim 160 \mathrm{~K}$ suggest either a broad fluctuation region associated with the transition at $T_{\mathrm{c} 1}$ or another incipient transition that is eventually suppressed by the hidden electronic order. Further structure-sensitive experiments focusing towards small wavevectors could possibly shine more light on this issue. 


\section{ACKNOWLEDGMENTS}

The authors acknowledge the financial support of Slovenian Research Agency (research core funding NoP1-0040) and European Research Council Advanced Grant TRAJECTORY (GA 320602) for financial support, valuable discussions with V.V. Kabanov, Y.A. Gerasimenko and I.V. Madan and A. Meden for the initial characterization of molybdenum suboxide phases. JM acknowledges support by Program P1-0044 of Slovenian Research Agency.

\section{APPENDIX}

In Fig. 11 we show decomposition of the full DECP fit (Eq. 5) into different components. The sum of the fitted oscillator components at $T=10 \mathrm{~K}$ consisting from oscillators O1-O4 is shown in Fig. 11 a) in comparison to the total transient reflectivity. Note that the displacive driven oscillator coordinates contain both, an oscillating

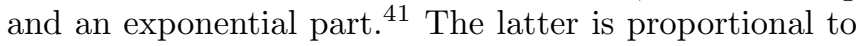
the exponential displacive drive and relaxes with the time constant $\tau_{\text {disp. }}$.

The exponential components with the remaining oscillators are clearly revealed after we subtract the four fitted oscillator responses [see Fig. 11 b)]. The non-oscillating part of the residue can not be completely fit with a two component exponential relaxation $\frac{54}{1}$ indicating the presence of picosecond component e1.
* Venera.Nasretdinova@ijs.si

1 P. Fiordiponti, G. Pistoia, C. Temperoni, M. Icovi, and S. Panero, J. Electroanal. Chem. 108, 181 (1980)

2 C. Julien, A. Mauger, A. Vijh, and K. Zaghib, Lithium batteries (Springer, 2015)

3 H.-S. Kim, J. B. Cook, H. Lin, J. S. Ko, S. H. Tolbert, V. Ozolins, and B. Dunn, Nat. Mater. 16, 454 (2016)

${ }^{4}$ S. Barabash, T. Chiang, and D. Pramanik, "Moox-based resistance switching materials," (Dec 2014), www.google. com/patents/US8907314

M. Borovšak, P. Sutar, E. Goreshnik, and D. Mihailovic, Applied Surface Science 354, 256 (2015)

6 M. Sato, H. Fujishita, S. Sato, and S. Hoshino, Journal of Physics C: Solid State Physics 19, 3059 (1986)

${ }^{7}$ H. Fujishita, M. Sato, S. Shapiro, and S. Hoshino, Physica B+C 143, 201 (1986), ISSN 0378-4363

${ }^{\circ}$ H. Fujishita, M. Sato, S. Sato, and S. Hoshino, J. Solid State Chem. 66, 40 (1987)

9 A. J. H. Komdeur, J. L. de Boer, and S. van Smaalen, J. Phys.: Cond. Matter 2, 45 (1991)

${ }_{10}$ A. Fang, N. Ru, I. R. Fisher, and A. Kapitulnik, Phys. Rev. Lett. 99, 046401 (Jul 2007), https://link.aps.org/doi/ 10.1103/PhysRevLett.99.046401

11 J. Axe, M. Iizumi, and G. Shirane, "Materials," (NothHolland, Amsterdam, 1986) Chap. 10, pp. 1-48

12 P. Bak, Reports on Progress in Physics 45, 587 (1982), http://stacks.iop.org/0034-4885/45/i=6/a=001

13 J.-P. Pouget, Low-Dimensional Electronic Properties of Moly-bdenum Bronzes and Oxi-des (ed. C. Schlenker) (Kluwer Academic Press, Dordrecht, 1989)

14 E. Canadell and M.-H. Whangbo, Inorg. Chem. 29, 2256 (1990)

15 H. Sowa, W. Steurer, and J. L. D. Boer, Phase Transitions 47, 1 (1994)

16 G. A. Samara, Comments Sol. State Phys 8 (1977)

17 G. Samara and P. Peercy (Academic Press, 1982) pp. 1 118, http://www.sciencedirect.com/science/article/ pii/S0081194708601149

18 P. Monceau, Adv. Phys. 61, 325 (2012)

19 R. Yomo, K. Yamaya, M. Abliz, M. Hedo, and Y. Uwatoko, Phys. Rev. B 71, 132508 (Apr 2005), https://link.aps.

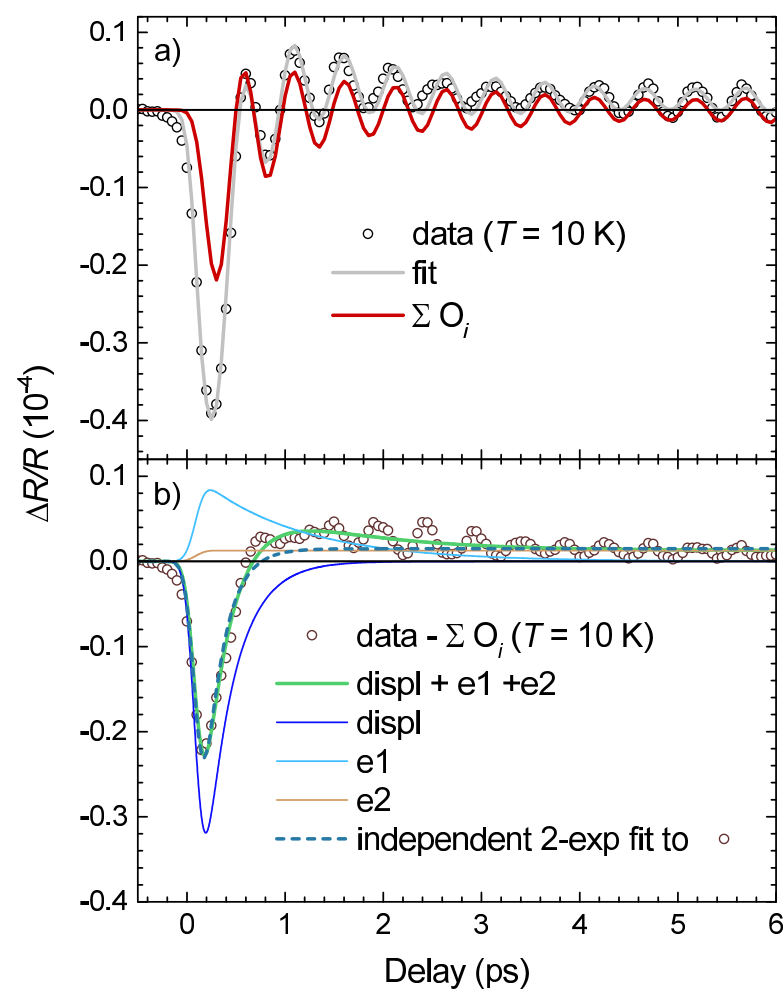

Figure 11. Decomposition of the transient reflectivity at $10 \mathrm{~K}$ into components. a) Comparison of the raw transient reflectivity at $10 \mathrm{~K}$, the fit (Eq. 5) and the sum of the four fit oscillatory components, O1-O4. b) Comparison of the residual of the raw data (with O1-O4 components subtracted) to the exponential fit components. It is evident that the sum of components displ, e1 and e2 fits the residual better than a simple two exponent fit.

org/doi/10.1103/PhysRevB.71.132508

${ }^{20}$ R. L. Smith and G. S. Rohrer, Journal of Solid State Chem- 
istry 124, 104 (1996)

21 V. Nasretdinova, Y. Gerasimenko, J. Mravlje, P. Sutar, D. Svetin, G.-M. Gatti, A. Y. Kuntsevich, M. Grioni, and D. Mihailovic(2018), to be published

22 F. Weber, S. Rosenkranz, J.-P. Castellan, R. Osborn, G. Karapetrov, R. Hott, R. Heid, K.-P. Bohnen, and A. Alatas, Phys. Rev. Lett. 107, 266401 (Dec 2011), https://link.aps.org/doi/10.1103/PhysRevLett.107. 266401

23 J. Demsar, K. Biljaković, and D. Mihailovic, Phys. Rev. Lett. 83, 800 (1999)

24 J. Demsar, V. K. Thorsmølle, J. L. Sarrao, and A. J. Taylor, Phys. Rev. Lett. 96, 037401 (Jan 2006), https: //link.aps.org/doi/10.1103/PhysRevLett.96.037401

${ }^{20}$ R. Yusupov, T. Mertelj, V. V. Kabanov, S. Brazovskii, P. Kusar, J.-H. C. Chu, I. R. Fisher, and D. Mihailovic, Nature Physics 6, 681 (2010)

26 T. Mertelj, V. V. Kabanov, C. Gadermaier, N. D. Zhigadlo, S. Katrych, J. Karpinski, and D. Mihailovic, Phys. Rev. Lett. 102, 117002 (Mar 2009), https://link.aps. org/doi/10.1103/PhysRevLett.102.117002

J. Demsar, L. Forro, H. Berger, and D. Mihailovic, Phys. Rev. B 66, 041101 (2002)

${ }^{28}$ Y. Toda, T. Mertelj, T. Naito, and D. Mihailovic, Phys. Rev. Lett. 107, 227002 (Nov 2011), https://link.aps . org/doi/10.1103/PhysRevLett.107.227002

${ }^{29}$ J. W. Harter, D. M. Kennes, H. Chu, A. de la Torre, Z. Y. Zhao, J.-Q. Yan, D. G. Mandrus, A. J. Millis, and D. Hsieh, Phys. Rev. Lett. 120, 047601 (Jan 2018), https://link. aps.org/doi/10.1103/PhysRevLett.120.047601

30 K. Ishioka, A. K. Basak, and H. Petek, Physical Review B 84, 235202 (2011)

31 Y. Toda, F. Kawanokami, T. Kurosawa, M. Oda, I. Madan, T. Mertelj, V. V. Kabanov, and D. Mihailovic, Phys. Rev. B 90, 094513 (Sep 2014)

32 S. Tsuchiya, Y. Sugawara, S. Tanda, and Y. Toda, Journal of Optics 17, 085501 (2015), http://stacks .iop.org/ $2040-8986 / 17 / i=8 / a=085501$

33 K. Nakagawa, S. Tsuchiya, J. Yamada, and Y. Toda, Journal of Superconductivity and Novel Magnetism 29, 3065 (Dec 2016), ISSN 1557-1947, https://doi.org/10.1007/ s10948-016-3741-6

34 S. Tsuchiya, K. Nakagawa, J. Yamada, H. Taniguchi, and Y. Toda, Phys. Rev. B 96, 134311 (Oct 2017), https:// link.aps.org/doi/10.1103/PhysRevB.96.134311

35 O. Bertrand, N. Floquet, and D. Jacquot, J. Cryst. Growth 96, 708 (1989)

36 L. Stojchevska, M. Borovšak, P. Foury-Leylekian, J.-P. Pouget, T. Mertelj, and D. Mihailovic, Phys. Rev. B 96, 035429 (2017)
37 A. Yariv and P. Yeh, Optical Waves in Crystals Propagation and Control of Laser Radiation (Wiley, 2002)

38 E. Koch, A.Otto, and K. Kliewer, Chem. Phys. 3, 362 (1974)

39 R. V. Yusupov, T. Mertelj, J. H. Chu, I. R. Fisher, and D. Mihailovic, Phys. Rev. Lett. 101, 246402 (2008)

40 M. Borovšak, L. Stojchevska, P. Sutar, T. Mertelj, and D. Mihailovic, Physical Review B 93, 125123 (2016)

${ }^{41}$ H. J. Zeiger, J. Vidal, T. K. Cheng, E. P. Ippen, G. Dresselhaus, and M. S. Dresselhaus, Physical Review B 45, 768 (1992)

42 M. I. Aroyo, J. Perez-Mato, D. Orobengoa, E. Tasci, G. De La Flor, and A. Kirov, Bulg. Chem. Commun 43, 183 (2011)

43 A. Rothwarf and B. N. Taylor, Phys. Rev. Lett. 19, 27 (Jul 1967), https://link.aps.org/doi/10.1103/ PhysRevLett.19.27

${ }^{44}$ V. V. Kabanov, J. Demsar, B. Podobnik, and D. Mihailovic, Phys Rev. B 59, 1497 (1999)

45 Y. Toda, T. Mertelj, P. Kusar, T. Kurosawa, M. Oda, M. Ido, and D. Mihailovic, Phys. Rev. B 84, 174516 (Nov 2011), https://link.aps.org/doi/10.1103/PhysRevB. 84.174516

46 To fit the finite $A_{\text {displ }}$ above $T_{\text {IC }}$ in the case of $T$-dependent gap we assume a small $T$-independent background.

47 It is conceivable that the displacive component can not be completely described by an exponential fit and the displacive component residual is picked by component e1.

48 Similar tendency is also found in the Raman mode width, albeit proximity to the notch-filter cutoff prevents reliable linewidth temperature determination

49 H. Schaefer, V. V. Kabanov, and J. Demsar, Physical Review B 89, 045106 (2014)

50 Except the 2.6- $\mathrm{THz}$ mode that is rather weak in both, RS and the coherent response.

51 The coupling has to be weak since no significant broadening of the modes is observed despite significant overlap with mode $\mathrm{O} 1$ at low $T$.

${ }^{52}$ Y. Zhang, C. Wang, G. Liu, A. Liang, L. Zhao, J. Huang, Q. Gao, B. Shen, J. Liu, C. Hu, W. Zhao, G. Chen, X. Jia, L. Yu, L. Zhao, S. He, F. Zhang, S. Zhang, F. Yang, Z. Wang, Q. Peng, Z. Xu, C. Chen, and X. Zhou, Science Bulletin 62, 950 (2017), ISSN 2095-9273, http://www.sciencedirect. com/science/article/pii/S2095927317302918

53 H. Chi, C. Zhang, G. Gu, D. E. Kharzeev, X. Dai, and Q. Li, New Journal of Physics 19, 015005 (2017), http: //stacks . iop.org/1367-2630/19/i=1/a=015005

${ }^{54}$ In order to fit the sub-picosecond relaxation and the longdelay offset at least two exponential components are necessary. One with $\tau \sim \tau_{\text {disp }}$ and one with $\tau \rightarrow \infty$. 